\title{
REPORT ON THE PROGRESS \\ IN STELLAR EVOLUTION TO 1950
}

\author{
OWEN GINGERICH \\ Harvard-Smithsonian Center for Astrophysics \\ 60 Garden Street, Cambridge, MA 02138, U.S.A \\ (ginger@cfa.harvard.edu)
}

At a joint meeting of the American Astronomical Society and the American Physical Society held in June of 1940, the University of Michigan astronomer Dean McLaughlin (1940) gave a review of the current understanding of stellar evolution. At the end he somewhat facetiously remarked that, "For several years I have told students that I knew all about stellar evolution in 1923, less is 1925, and nothing at all since 1930." I would like to suggest that those dates were not chosen randomly, and in the first part of my own survey of stellar evolution to 1950 , I would like to explain the significance of those dates.

First, some background for understanding the status of stellar evolution studies in the early 1920s. The subject was then scarcely more than fifty years old. If that surprises you, remember that there are two critical elements required before it makes any sense even to talk about stellar evolution. One is a time scale far in excess of the 6,000 years assumed for many centuries in Christendom. The other is the fundamental idea of conservation of energy. Both of these concepts emerged by the middle of the nineteenth century, the work of geologists and physicists respectively, and almost immediately Hermann von Helmholtz and Sir William Thompson (later Lord Kelvin) recognized that the source of the sun's energy posed a critical problem. In a series of papers beginning in 1854 Thompson concluded that gravitational contraction offered the only reasonable physical explanation for the sun's prodigious luminous output, and that the sun's age was probably not in excess of 100 million years. This brought him into confrontation with Charles Darwin, who had implied a timetable of some billions of years in the first edition of his On the Origin of Species (Darwin, 
1859$, p.287 $)^{1}$. The tension between the geologists and physicists remained unresolved, and in 1892 Thompson repeated the exact words he had used almost forty years before:

"Within a finite period of time past the earth must have been, and within a finite period of time to come must again be, unfit for the habitation of man as at present constituted, unless operations have been and are to be performed which are impossible under the laws going on at present in the material world." (Lord Kelvin, 1892).

The solution of Kelvin's dilemma lay in the discovery of sub-atomic energy, as it was called in those days, and led to a memorable moment described by Ernest Rutherford. Rutherford had concluded that energy emitted by radium suggested another energy source, and that the world could be much older than Kelvin had argued. He advanced these views in a Friday Evening Discourse at the Royal Institution in 1904. Rutherford's own account is characteristic:

"I came into the room, which was half dark, and presently spotted Lord Kelvin in the audience and realized that I was in for trouble at the last part of my speech dealing with the age of the earth where my views conflicted with his. To my relief Kelvin fell fast asleep but, as I came to the important point, I saw the old bird sit up, open an eye and cock a baleful glance at me! Then a sudden inspiration came and I said that Lord Kelvin had limited the age of the earth provided no new source was discovered. "That prophetic utterance refers to what we are considering tonight, radium!" Behold! the old boy beamed upon me!" (Eve, 1939, p. 107).

Granted a long time scale, and the conservation of mass-energy, studies of stellar evolution require a knowledge of stellar structure and of energy sources, which in turn demand information about chemical compositions, and these rest on an observational base. The lack of appropriate observations, of a sound physical theory of internal constitutions of stars, or of knowledge of atomic and nuclear structure crippled early progress in understanding stellar evolution, but this did not deter speculation about the life histories of stars.

Thus, just as soon as it was recognized that stars fell into a relatively small number of spectral categories, Norman Lockyer came forward with an influential theory of stellar evolution to account for the pattern of hot and cool stars. In the 1880s he proposed his famous "temperature arch" in which stars began as immense swarms of metcuritic particles undergoing gravitational contraction. With rising temperatures, giant red $\mathrm{M}$ stars such as Antares would form, followed by further contraction and temperature

\footnotetext{
1 "The denunciation the Weald must have required $306,662,400$ years, or say three hundred million years... So that in all probability a far longer period than 300 million years has elapsed since the latter part of the Secondary period."
} 


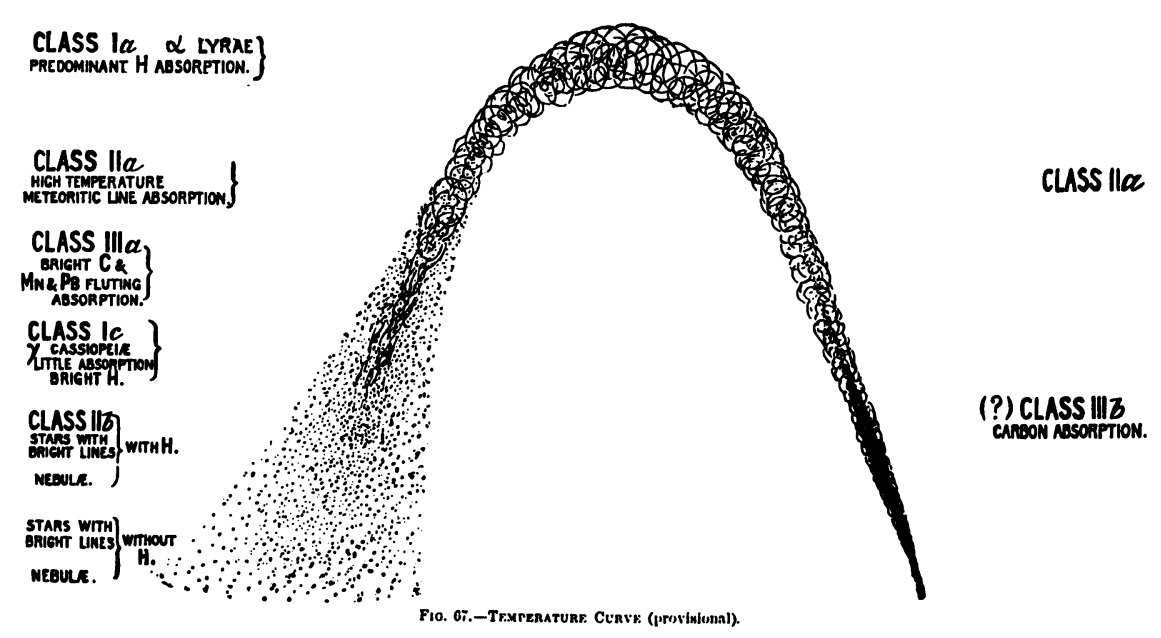

Figure 1. Lockyer's temperature arch, from his The Meteoritic Hypothesis (Lockyer, 1890, p. 375)

increase that would in sequence produce K, F, A, and B spectra. At the top of the arch hot, true stars would form, which would gradually cool back down to $\mathrm{K}$ and $\mathrm{N}$ stars.

In fact, most of Lockyer's contemporaries preferred a more linear evolutionary interpretation of the spectral sequence, which has left its vestiges in the expressions "early-type" and "late-type" stars. However, in 1914, Henry Norris Russell in introducing his spectral class-luminosity diagram with its dwarf and giant sequences noted that the stellar densities increased from the giant branch down the dwarf sequence, that is, what we now call the main sequence. "If that is also the order of advancing age, we are led at once back to Lockyer's hypothesis that a star is hottest near the middle of its history, and that the redder stars fall into two groups, one of rising and the other of falling temperature," Russell wrote. "The giant stars then represent successive stages in the heating up of a body, and must be more primitive the redder they are; the dwarf stars represent successive stages in later cooling, and the reddest of these are the farthest advanced." (Russell, 1914).

Among those who endorsed this view was Arthur Eddington, who remarked in the Observatory that ${ }^{2}$ "the most attractive part of the [theory] is the explanation in terms of the Lane-Ritter theory of the evolution

\footnotetext{
${ }^{2}$ What Eddington here referred to is the earlier, and originally rather disconcerting, result obtained by Lane and Ritter that if a star loses energy and contracts, its interior must heat up.
} 
of stars." Russell assumed that the giant stars were contracting and heating up, whereas when they reached the main sequence the contraction ceased and they merely cooled off, descending down the sequence.

In general spectroscopists were reluctant to subscribe to this view; everyone believed that stars would originate from nebulosity, but the close link between $\mathrm{O}$ and $\mathrm{B}$ type spectra and the spectra of planetary nebulae suggested that $M$ giant stars were not a satisfactory starting point. But in his Astronomical Physics textbook written in 1924, F.J.M. Stratton (p. 179) described a compromise view with two possible evolutionary sequences, one beginning with the hottest O-B-A stars and cooling down the main sequence, the other in the giant branch down the main sequence, either M-K-G-F or N-R-G-F. "To sum up the views advanced [here]," he wrote, "the evidence points to two sets of massive stars in an early stage of evolution. Two separate sources are indicated, namely, diffuse nebulosity and spiral nebulae. It is suggested that planetary nebulae, $O$ and $B$ type giants come from diffuse nebulosity, while $M$ and $N$ giants come from condensations in the arms of spiral nebulae" (Stratton, 1925). To make sense of this passage, we must note that Stratton was much influenced by James Jeans' Problems in Cosmogony, in which Jeans assumed that spiral nebulae were relatively small objects within the Milky Way, and possibly solar systems in formation.

This, then, was the received view around 1923 when Dean McLaughlin thought he understood everything about stellar evolution. What had happened by 1925 that made McLaughlin less sure that he understood stellar evolution? The most notable intervening event was Eddington's publication of a series of papers that would soon lead to his great classic, The Internal Constitution of the Stars. In the August 8, 1925 issue of Nature, Henry Norris Russell summarized the Lockyer theory of stars of rising and falling temperatures. "The recognition of the sequences of giant and dwarf stars lent strong support to this theory, and as the present writer showed eleven years ago a great mass of observed details fits in with remarkable completeness with the idea that the stars rise in temperature until the gas in their interior becomes compressive only with difficulty, and then cool down again" (Russell, 1925a). But then, citing primarily Eddington's recent work on stellar interiors, he conceded that, "The feature of the older theory which assumed a fall of internal temperature in the denser dwarf stars owing to the close-packing of atoms, must finally be abandoned."

Writing more popularly in Scientific American two months later, Russell used his temperature-luminosity diagram to illustrate an evolutionary track in which giant stars condensed and moved to the main sequence, worked their way down by mass exhaustion, and finally swung left into the white dwarf region. The caption proclaimed that, "The new scheme of stellar 


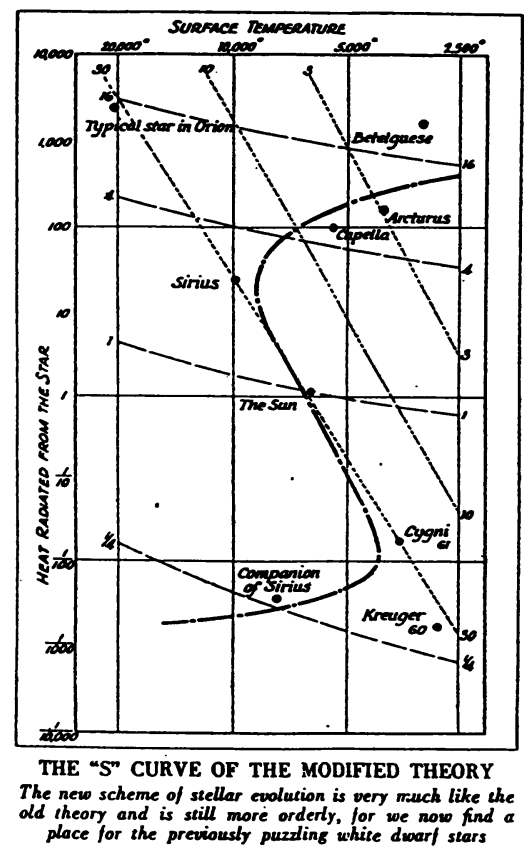

Figure 2. Russell's modified stellar evolution theory (Russell, 1925a)

evolution is very much like the old theory and is still more orderly, for we now find a place for the previously puzzling white dwarf stars" (Russell, 1925b).

The following year, 1926, brought the publication of Eddington's The Internal Constitution of the Stars, which summed up the work he had begun already in 1917. In a lucid and succinct review of this achievement, T.G. Cowling (1966) notes that Eddington's initial assumptions, in addition to the usual ones of a perfect gas and uniform composition, were those of radiative transfer and partial support by radiation pressure. He also assumed constant opacity and a constant rate of generation of energy per unit mass. Integrating the three resulting differential equations subject to the boundary conditions that the pressures must become negligible at the surface and $M(0)=0$, there remains one constant of integration. To evaluate this unknown, Eddington chose a mean molecular weight of 2.1, corresponding to a highly ionized mixture of terrestrial composition, that is, with an insignificant contribution from hydrogen. The great triumph of this calculation was the prediction of a mass-luminosity relation close to the 


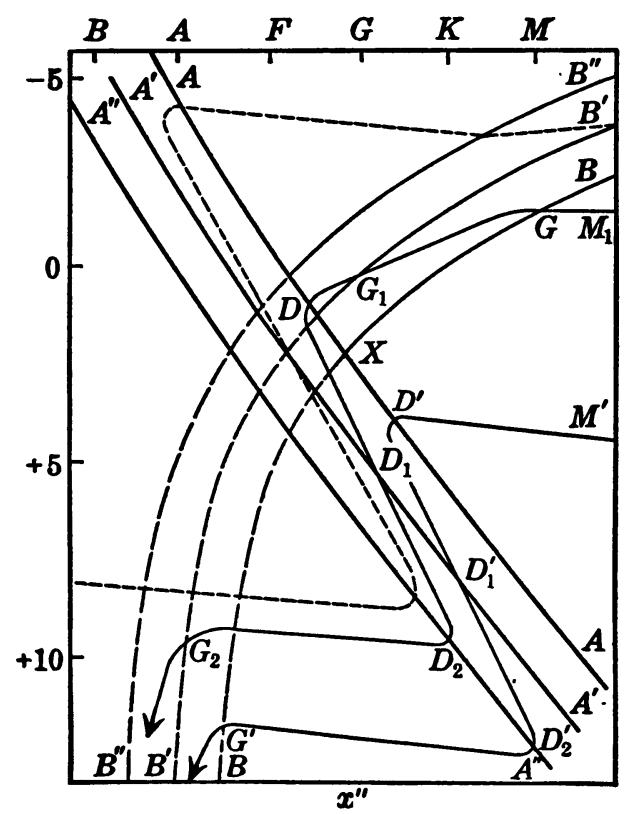

Fig. 307. Stellar Evolution

Figure 3. Russell's textbook diagram of stellar evolution (Russell et al., 1927, p. 911)

observational results, although to make the fit better he had to increase the opacity a factor of ten higher than given by Kramers' theory. As Cowling remarks, one cannot help but feel that he was lucky.

Eddington's mass-luminosity relation essentially destroyed Russell's earlier evolutionary theory, for it showed that the fundamental difference in stars going down the main sequence was that they had decreasing mass (see Eisberg, 1991). Eddington himself found it difficult to give up this evolutionary scheme because it had played an important role in the construction and presentation of his stellar model, and even in his book he produced a long discussion of stellar energy and evolution dependent upon a subatomic process of electron-proton annihilation that would allow stars to lose mass as they migrated down the dwarf sequence.

Russell himself responded to Eddington's findings in the brilliant textbook that he published with R.S. Dugan and J.S. Stewart in 1927, where he repeated the modifications he had introduced in 1925. In describing the older theories, he remarked that

"This scheme was suggested by Sir Norman Lockyer, who called attention to the importance of distinguishing between young stars of rising temperature and older ones which were cooling. It was advocated and extended, in 1913, 
by Russell, who pointed out that the giant and dwarf stars (which had then recently become known) presented exactly the characteristics which should be exhibited by stars of rising and falling temperature....

This theory represented almost all observed facts, not only in a general way, but in detail....

We know now, however, that this assumption about the properties of the gas [that it would be just at the stage of difficult compressibility when the maximum temperature was reached] is certainly wrong, though no one would have supposed this in 1913. The theory, moreover, fails entirely to account for the white dwarfs" (Russell et al., 1927, p. 909).

Russell's revised theory, somewhat embellished from what he had recently depicted in Scientific American, must be seen to be believed. The figure shows an $\mathrm{H}-\mathrm{R}$ diagram with crossed highways, one, matching the main sequence, labeled with D's, and the other partially matching the giant branch but continuing beyond it across the main sequence, labeled with G's. Russell knew from Eddington's Internal Constitution of the Stars that an intense central energy source was indicated. Arguing carefully through what is now commonly called the Russell-Vogt theorem, that mass and composition alone situate the stars in their own peculiar places on the diagram, he went on to conclude that the stars had different mixtures of energy-rich active material called dwarf-stuff and giant-stuff. A massive star well endowed with giant-stuff would find a stasis in the giant region of the diagram, and only when that material was exhausted would it move by gravitational contraction to the main sequence where it could continue to burn even more hotly with the remaining active dwarf-stuff. A star of much less mass would apparently burn its dwarf-stuff first.

Even more interesting evolutionary tracks appear in Russell's second diagram. Perhaps the exhaustion of the giant-stuff or the dwarf-stuff would entail a mass loss. Russell makes the calculation by Einstein's $E=m c^{2}$ equation without mentioning it explicitly. In conclusion he notes that "Various other cases can easily be worked out." At the time Russell's proposal seemed plausible if incomplete, and though it was soon enough abandoned, its legacy was the notion that different luminosity classes might in fact be powered by different processes.

Given the speculative nature and obvious incompleteness of Russell's proposal, it is little wonder that McLaughlin was much less sure that he understood stellar evolution in 1925 than he had been in 1923. But why did he claim to understand nothing at all in 1930?

By 1930 it was more apparent that Eddington's opacity assumptions were in trouble, and the solution might somehow be found in the mysterious quantum mechanics that was so profoundly changing the face of physics. But there was something more, namely, that the chemical compo- 


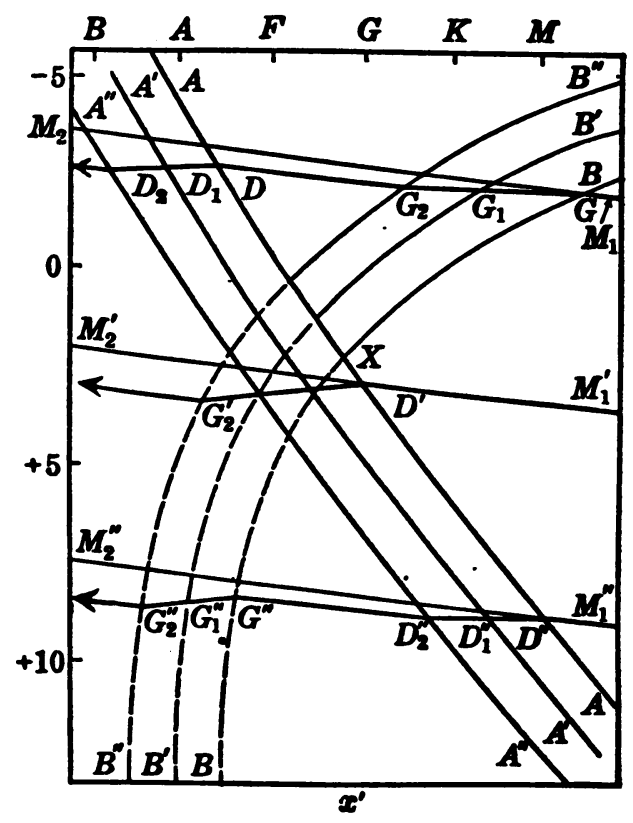

Frg. 306. Stellar Evolution

Figure 4. Russell's second textbook diagram (Russell et al., 1927, p. 916)

sition of the stars might not be primarily iron after all, despite the high iron abundance in meteorites and presumably in the earth's core. It was as if one of the fundamental principles of astronomy, the uniformity of nature's laws throughout the cosmos, was crumbling.

In 1925, in what Otto Struve called the most brilliant $\mathrm{PhD}$ thesis ever written in astronomy, Cecilia Payne essentially solved one of the outstanding problems of the day, the determination of stellar temperatures. In fact, she solved it so successfully that it more or less became a non-problem, and her thesis is primarily cited today for a chapter near the end that opened a whole new area, the question of the relative abundance of the elements. In her research she got the first glimpse of the possibility of a high hydrogen abundance. But while the numbers seemed to show that hydrogen was a million times more abundant than iron, she stated that, "The outstanding discrepancies between the astrophysical and terrestrial abundances are displayed for hydrogen and helium. The enormous abundance derived for these elements in the stellar atmosphere is almost certainly not real. Probably the result may be considered, for hydrogen, as another aspect of its abnormal behavior" (Payne, 1925, pp. 188-89).

Some years ago I asked Professor Payne-Gaposchkin why she had con- 
cluded that the enormous hydrogen abundance was not real, and she replied that no doubt Henry Norris Russell had talked her out of it. Indeed, here is the "smoking gun" letter"

January 14, 1925.

My dear Miss Payne:

Here, at last, are your notes on relative abundance which you were so good as to send me some time ago....

You have some very striking results which appear to me, in general, to be remarkably consistent. Several of the apparent discrepancies can be easily cleared up. [Here Russell discusses $\mathrm{Mg}, \mathrm{Mg}+$, and $\mathrm{K}$ in some detail.]

There remains one very much more serious discrepancy, namely, that for hydrogen, helium and oxygen. Here I am convinced that there is something seriously wrong with the present theory. It is clearly impossible that hydrogen should be a million times more abundant than the metals, and I have no doubt that the number of hydrogen atoms in the two quantum state is enormously greater than is indicated by the theory of Fowler and Milne. Compton and I sent a little note to 'Nature' about metastable states, which may help to explain the difficulty....

Very sincerely yours, Henry Norris Russell

It was not until four years later that Russell changed his mind, and in an Astrophysical Journal paper entitled "On the Composition of the Sun's Atmosphere", conceded that a high abundance of hydrogen helped to explain a number of previously puzzling astronomical facts (Russell, 1929). Part of his argument rested on the results published by Albrecht Unsöld the previous year, in 1928. Unsöld had used the observed contours of resonance lines to determine the number of atoms involved in the production of the line, and had concluded that hydrogen was a million times more abundant than the metals.

A number of years ago I asked Unsöld if he was not one of the first to appreciate the high cosmic abundance of hydrogen. "Quite the contrary.", he replied, "The spectroscopic results only concerned the solar atmosphere, and there was no reason to believe that it applied to the entire sun." ${ }^{4}$ Indeed, since hydrogen was the lightest element, most astronomers probably assumed that it had naturally floated to the surface of the sun.

\footnotetext{
${ }^{3}$ Henry Norris Russell Papers, Series I: Outgoing Correspondence, Box 9, Folder 37; published with permission of the Manuscipt Division, Department of Rare Books and Special Collections, Princeton University Libraries.

${ }^{4}$ Paraphrased from an oral history interview, 1978, on deposit at the Niels Bohr Library of the American Institute of Physics.
} 
In recent years there has been an increasing tendency to attribute the discovery of the cosmic hydrogen abundance to Cecilia Payne. The historical record makes this attribution dubious. In the first place, she, and the advisors she trusted, all found the notion of the uniformity of nature and nature's laws compelling, and it seemed reasonable then to believe that terrestrial abundances mirrored those elsewhere in the cosmos. This made an enormous solar hydrogen abundance highly anomalous. Secondly, there were not necessarily grounds for extrapolating a high hydrogen abundance found in stellar atmospheres to stellar interiors.

Eddington, who had, much to Miss Payne's delight, commented in his Internal Constitutions that her procedure for determining the abundances "is not so wild as we might suppose at first," (Eddington, 1926, p. 369) finally in desperation began to consider a high hydrogen abundance for an entire star as a way to resolve the opacity discrepancy. As he was completing his analysis, in March of 1932, he received a manuscript from Bengt Strömgren, who had from a different approach also produced a stellar interior model based on a high hydrogen abundance (Strömgren, 1932; Eddington, 1932). In reply, Eddington wrote:

"My results agree with yours almost exactly. I shall not be deterred from publishing my results, however; nowadays for two investigators to agree is such a rare phenomenon that it deserves to be recorded!" 5 As historian Karl Hufbauer has written, "This agreement settled the matter so far as Eddington, Strömgren, and most other theoretical astrophysicists were concerned. Thenceforth, they regarded hydrogen as the predominant constituent not only on but in the stars obeying the mass-luminosity relation."

Meanwhile, on the stellar energy front, congenial new reasons emerged for accepting a high hydrogen abundance. In 1928 George Gamow had developed a quantum-mechanical theory for the penetration of a nucleus by a fast-moving alpha particle. Robert d'Escourt Atkinson and Fritz G. Houtermans, who were working in Göttingen and who had consulted with Gamow on a skiing trip, applied and extended Gamow's theory to the nuclei within stars (Atkinson and Houtermans, 1929). By combining Gamow's penetration probability with a Maxwellian distribution of velocities, they provided an initial theory of nuclear energy generation within stars. In particular, they found that the most effective interactions involved light elements, and that only a few particles in the high-energy tail of the Maxwellian distribution would penetrate these nuclei.

At first Atkinson had trouble getting a hearing for his theory, in part because E.A. Milne had launched a heavy critique of Eddington's stellar models, proposing his own version with a highly condensed central core and

\footnotetext{
${ }^{5}$ Quoted from Karl Hufbauer, "Stellar Structure and Evolution", in Owen Gingerich (editor), The General History of Astronomy 4B (Cambridge, forthcoming)
} 
central temperatures in the billions of degrees, far higher than Atkinson's scheme required. In Cowling's assessment, Milne's work, if not altogether wrong, was completely premature (Cowling, 1966). Eddington ridiculed his solutions, once saying that they reminded him of a reputed Tripos question that began, "In an apple pie not more than 30 feet high...." But at least Milne's centrally-collapsed models, in the hands of S. Chandrasekhar, turned out to be relevant to an understanding of white dwarf stars.

Meanwhile, Atkinson took a physics position at Rutger's University in New Jersey, where he found a sympathetic audience in a near neighbor, Princeton's Henry Norris Russell. With Russell's encouragement, in a long and far-ranging article in the Astrophysical Journal, Atkinson (1931a,b) addressed the energy problem in much greater detail. Accepting the idea of a great stellar abundance of hydrogen, Atkinson considered how fusion of hydrogen into helium might provide the required energy for the sun and stars. Because he knew that a quadruple collision of four protons to form an alpha particle would be quite improbable, he suggested that successive protons could be absorbed by nuclei until they became unstable and ejected alpha particles.

It was the great flowering of nuclear physics in the 1930s that made it possible to find specific solution of the sort envisioned by Atkinson. The discovery of a wide variety of nuclear reactions, together with information about the stability of various isotopes, opened up interesting questions that no one had even thought to frame before. For example, as Ernest Rutherford wrote in 1937, "When our knowledge of transformations is more advanced, we may be able to understand the reason of the relative abundance of different elements in the earth and why, on average, even-numbered elements are far more abundant than odd-numbered elements. We thus see how the progress of modern alchemy will not only add greatly to our knowledge of the elements, but also of their relative abundance in our universe" (Rutherford, 1937, p. 67).

In Germany, C.F. von Weizsäcker considered not only how hydrogen might have powered the sun for billions of years, but also how the heavier elements could have been built up by such reactions. By 1937 he had proposed that a series of reactions, beginning with the proton-proton production of deuterium, could have led to the heavier elements, the so-called Aufbau hypothesis. By the following year he recognized the inherent difficulties posed for his scheme by the apparent absence of stable mass 5 and 8 isotopes, and out of the relatively few remaining options, he hit upon the carbon-nitrogen cycle.

Meanwhile, George Gamow as well as many other refugees from central Europe had emigrated to America. Continually interested in such problems, Gamow acted as a gadfly in a long correspondence with Chandrasekhar and 
to stir up the interest of John von Neumann, Hans Bethe, and his secondin-command at the George Washington University in Washington, Edward Teller. Gamow regularly published short notes in the Physical Review and elsewhere; for example, speaking at an Ohio State University symposium in 1935, he described the physical state of matter deep inside stars where "all atoms are completely ionized, all electronic shells being torn away from the nuclei by the violent collisions." He went on to say, "we are interested especially in the role played by stellar nuclei in the formation of heavy elements," and he expressed the hope that "further investigation will clarify the relative importance of various processes and lead to a complete explanation of the relative abundance of different elements in the universe" (Gamow, 1935).

In that same year he and Teller, together with Merle Tuve from the Carnegie Institution in Washington, began a series of short annual conferences on theoretical physics. In March of 1938 the topic was stellar energy, and Gamow persuaded Hans Bethe to come, along with other physicists and astronomers including Chandrasekhar, Strömgren, and Menzel. Decisive results regarding the lack of stable mass 5 and 8 isotopes were reported, and the focus was shifted to the stability of $\mathrm{Be}-6$ and the production of deuterons by a primary process involving two protons. There were also reports on the stellar opacity problem, and on a stellar model with a highly condensed neutron core (Chandrasekhar et al., 1938; Gamow and Tuve, 1938).

The most important consequence of the conference, however, was its catalytic effect on Hans Bethe, who, in the words of one participant, had come "knowing everything about the interior of the atom and nothing about the interior of a star." Or, as Bethe himself put it in his Nobel prize lecture, "the astrophysicists told us physicists what they knew about the internal constitution of stars. This was quite a lot, and all their results had been derived without knowledge of the specific source of energy. The only assumption they made was that most of the energy was produced 'near' the center of the star" (Bethe, 1958). Bethe went on to say:

"Stimulated by the Washington Conference ... I examined the reactions between protons and other nuclei, going up in the periodic system (Bethe, 1939). Reactions between $H$ and He4 lead nowhere, there being no stable nucleus of mass 5. Reactions of $H$ with $L i, B e$, and B, as well as with deuterons, are all very fast at the central temperature of the sun, but just this speed of reaction rules them out: The partner of $H$ is very quickly used up in the process. In fact, and just because of this reason, all the elements mentioned, from deuterium to boron, are extremely rare on earth and in the stars, and can therefore not be important sources of energy.

The next element, carbon, behaves quite differently. In the first place, it is 

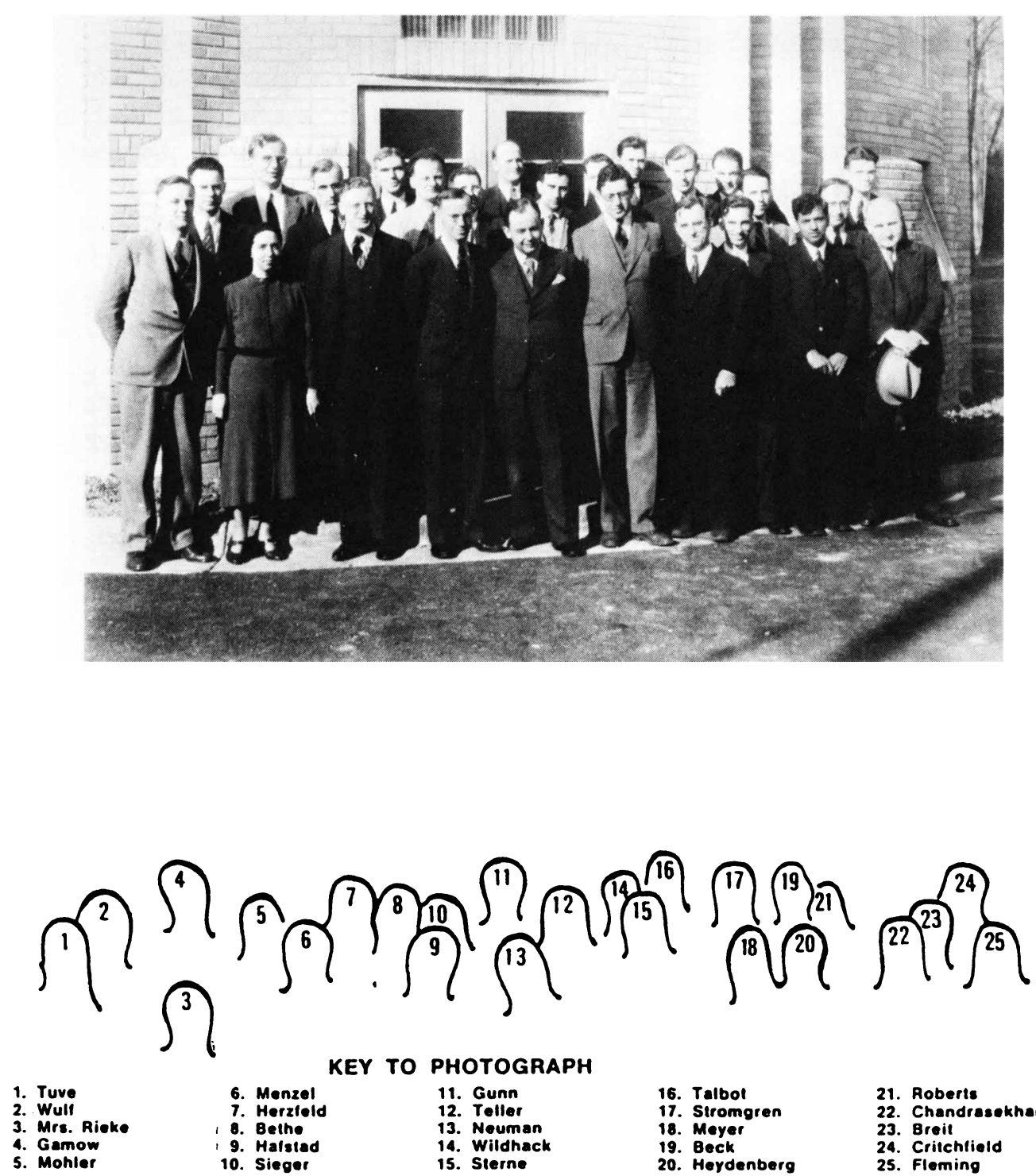

KEY TO PHOTOGRAPH

11. Gunn
12. Teller
13. Neuman
14. Wildhack

17. Stromgren

18. Meyer

21. Roberts

22. Chandrasekhar

23. Breit

14. Wildhack
15. Sterne

19. Beck

24. Critchlield

25. Fleming

Figure 5. Participants at the 1938 Washington Conference on Theoretical Physics (courtesy Special Collections Division, George Washington University Library, Washington, D.C.; caption courtesy of Griffith Observer ) 


\section{Lieber Hans!}

Der grosse Mendeleev hat nie gedacht das man kan seine periodishe systemme ins Himme leksen. Aben dass ist so! Die sterve die am B, Be, Li and D (!) lehben sollen in Russole diagram die, an thouptrerie jarrallele, Strveifer
machen Tun ind die

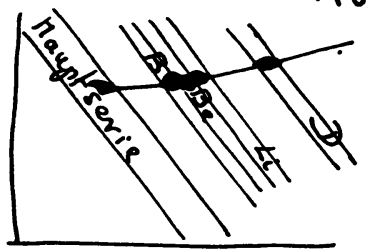
absoluten Grossen ron Giganton sehr soninutzig berannt and stalestisch kan man dass niont somen. För die Copheiden sind aher die Perioden deveocet gemesson and von secucutr-vaft abho"ngig. Also vehmen wird Gepherden!

Figure 6. Gamow to Hans Bethe, 11 March 1939, Division of Rare and Manuscript Collections, Carl A. Koch Library, Cornell University. "The great Mendeleev didn't know that one can learn his periodic system in the heavens. But it is so!"

an abundant element... Secondly, it undergoes a cycle of reactions...."

Unlike von Weizsäcker, Bethe made specific assumptions about the reaction rates in the carbon-nitrogen cycle, finding in 1938 that it gave about the correct energy production in the sun. By the time that McLaughlin wrote his review article in 1940 , the carbon-nitrogen cycle was not only well established, but the evolutionary patterns of stars were being rethought. According to Gamow, a star could enter the cool side of the giant branch and evolve rapidly to the main sequence, where the carbon-nitrogen cycle would be turned on, after which the star would slowly increase in luminosity until it used up its hydrogen. Would the star then rapidly become a white dwarf? Since Eddington had calculated that white dwarfs had about 
the same hydrogen content as main sequence stars, this scenario posed a problem. Thus, McLaughlin expressed his reservations about a complete endorsement of Gamow's views.

In fact, the problem of structure of white dwarfs and their place in the evolutionary scheme was a key topic in a conference held in Paris in July of 1939, on the eve of the Second World War. Many of the key players attended, including Atkinson, Walter Baade, Payne-Gaposchkin, Russell, and Strömgren. It was the scene for a replay of the infamous debate between Chandrasekhar and Eddington about whether or not massive stars could collapse without limit. Chandra pointed out that according to Sir Arthur, the four white dwarfs reported by Gerard Kuiper at the conference would have masses of $28,50,120$, and more than 190 solar masses, which he considered unreasonable if the white dwarfs were understood as part of a continuum of stars. Here I do not have time to recall some of the subsequent developments, such as Bethe and R.E. Marshak's work on the structure of white dwarfs, which led to the conclusion that hydrogen was absent in them (see Marshak, 1966) nor of Chandrasekhar's work with Mario Schönberg in the early 1940s, which placed an upper limit on the mass of a hydrogendepleted core.

In the early 1940s Gamow and his students worked on models with shell burning, in which the shell worked its way to the surface of the star, in contradiction to the Chandrasekhar-Schönberg limit. It was a battle of giants, with Gamow and Chandrasekhar and their students pitted against each other. The net effect of the 1940s decade was a standoff, leaving the evolutionary status of red giants essentially unexplained.

Both Chandrasekhar and Gamow turned to other interests, and in particular, Gamow turned to a cosmological theory for the origin of the chemical elements. Perhaps the most famous paper of that series is the one that appeared in the April First, 1948 issue of The Physical Review by Ralph Alpher, Bethe, and Gamow, which became known as the AlphaBeta-Gamma paper ${ }^{6}$. Gamow had been speculating that the various nuclear species were built up in a non-equilibrium process in the very early stages of the expanding universe, and Alpher had come looking for a doctoral thesis topic. Because electronic computers were still in an embryonic stage of development, Alpher divided the 238 atomic species into a dozen sections and averaged within the sections. The results seemed promising, and Bethe's name was added to the preliminary paper as part of an April Fool's Day prank. But more detailed calculations, published in 1950 by Alpher and R. Herman, revealed a fundamental difficulty caused by the absence of a stable mass 5 isotope (Alpher and Herman, 1950).

\footnotetext{
${ }^{6}$ See an account of the paper in Gamow, 1966, p. 127.
} 
Gamow was very frustrated by this result, and perhaps the best way to conclude a report on the progress in stellar evolution to the mid-century is to cite Gamow's "Cosmic Genesis" (Gamow, 1970). ${ }^{7}$ :

"In the beginning God created Radiation and Ylem (a mixture of protons and neutrons). And the Ylem was without shape or number, and the nucleons were rushing madly upon the face of the deep.

And God said: "Let there be mass two." And there was mass two. And God saw deuterium, and it was good.

And God said: "Let there be mass three." And there was mass three. And God saw tritium and tralphium, and they were good.

And God continued to call numbers until He came to the transuranium elements. But when He looked back on his work, He saw that it was not good. In the excitement of counting, He had missed calling for mass five, and so, naturally, no heavier elements could have been formed.

God was very disappointed by that slip and wanted to contract the universe again and start everything from the beginning. But that would be much too simple. Instead, being Almighty, God decided to make heavy elements in the most impossible way.

And so God said: "Let there be Hoyle." And there was Hoyle. And God saw Hoyle and told him to make heavy elements in any way he pleased.

And so Hoyle decided to make heavy elements in stars, and to spread them around by means of supernova explosions. But in doing so, Hoyle had to follow the blueprint of abundances which God prepared earlier when He had planned to make the elements from Ylem.

Thus, with the help of God, Hoyle made all heavy elements in stars, but it was so complicated that neither Hoyle, nor God, nor anybody else can now figure out exactly how it was done."

Gamow's whimsical parody, actually written in 1953, bridges into the developments of the 1950s. On the one hand, Fred Hoyle and his fellow steady-state cosmologists supposed the hydrogen was gradually created in the interstices of space to maintain a uniform density despite the continual expansion of the universe; they needed a way to make the heavy elements, presumably in the interiors of massive evolving stars. On the other hand, the detailed H-R diagrams for globular clusters being observed by Walter Baade's students and young proteges offered a challenge for detailed evolutionary calculations, because for the first time the red giant branch had been joined observationally with the main sequence. Allan Sandage, who had been working with Baade on the H-R diagram of M3, came to Princeton as a post-doc to work with Martin Schwarzschild on the calculation of models of stars with hydrogen-exhausted cores undergoing gravitation-

\footnotetext{
${ }^{7}$ Gamow speculated that this parady might account for his not having received an invitation to the 1958 Solvay Congress on cosmology.
} 
al contraction, these being a deliberate attempt to account for Sandage's thesis data. Their detailed calculations showed that as the core contracts, the envelope greatly expands, giving the giant stars high enough internal temperatures for nuclear processes to provide the required luminosities, but they were unable to explain the brighter features of the cluster's H-R diagram (Sandage and Schwarzschild, 1952).

Meanwhile, Ernst Öpik in 1951 and Edwin Salpeter independently in 1952 had shown that at sufficiently high temperatures carbon can be produced by triple helium collisions through the intermediary of unstable beryllium-8, and by 1954 Hoyle demonstrated that this triple alpha process could occur in red giant cores (Öpik, 1951; Salpeter, 1952; Hoyle, 1954). The following year Hoyle and Schwarzschild published their reconnaissance of stellar models all the way to the tip of the red giant branch, to the point soon to be called the helium flash (Hoyle and Schwarzschild, 1955). Schwarzschild quickly realized that at the this critical point the red giant star evolved more rapidly than the high- speed electronic computers of those days could calculate the changes. In any event, the rudiments of giant-star evolution had finally been delineated.

Let me reiterate what I said at the outset, that progress understanding stellar evolution requires not only a sound physical theory of internal constitutions and of nuclear structure, but also an appropriate and perceptive observational base, and it was especially the fresh observational insights of Walter Baade, his students, and those he inspired, that broke the evolutionary impasse to which astronomy had arrived in 1950.

Acknowledgments: I would especially like to thank Professor S.S. Schweber for sharing his highly relevant research materials with me, and also Karl Hufbauer, David DeVorkin, and Ralph Kenat for showing me the manuscripts of their forthcoming chapters in The General History of Astronomy 2B.

\section{References}

Alpher, R. and Herman, R., 1950, Rev. Mod. Phys. 22, 153

Atkinson, R.d'E., 1931a, Ap. J. 73, 250

Atkinson, R.d'E., 1931b, Ap. J. 73, 308

Atkinson, R.d'E. and Houtermans, F.G., 1929, Zeitschr.f. Physik 54, 656; also in Nature

Bethe, H.A., 1939, Phys. Rev. 55, 436

Bethe, H.A., 1958, Physics Today, September, 36

Burchfield, J.D., 1975, Lord Kelvin and the Age of the Earth, New York

Chandrasekhar, S., Gamow, G. and Tuve, M.A., 1938, Nature 141, 982

Cowling, T.G., 1966, Quart. J. R. A. S. 7, 121

Darwin, Charles, 1859, On the Origin of Species, London.

Eddington, A.S., 1926, Internal Constitution of the Stars, Cambridge

Eddington, A.S., 1932, M. N. R. A. S. 92, 471

Eisberg, J., 1991, Doctoral dissertation, Harvard University 
Eve, A.S., 1939, Rutherford, Cambridge

Gamow, G., 1935, The Ohio Journ. of Science, 35, 406

Gamow, G., 1966, Perspectives in Modern Physics: Essays in Honor of Hans A. Bethe, ed. R.E. Marshak, New York, p. 443

Gamow, G., 1970, My World Line: An Informal Biography, New York

Gamow, G. and Tuve, M.A., 1938, unpublished memorandum on the Fourth Washington Conference on Theoretical Pgysics, March 28, 1938, Chandrasekhas papers, University of Chicago Library

Hoyle, F., 1954, Ap. J. Suppl. 1, 121

Hoyle, F. and Schwarzschild, M., 1955, Ap. J. Suppl. 2, 1

Lord Kelvin, 1892, Fortnightly Review 57 (o.s.), March, 313. Quoted by Joe D. Burchfield (1975)

Lockyer, N. The Meteoritic Hypothesis, London

Marshak, R.E., 1966, Perspectives in Modern Physics: Essays in Honor of Hans A. Bethe, ed. R.E. Marshak, New York, p. 429

McLaughlin. D.B., 1940, P. A. S. P. 20, 358

Öpik, E., 1951, Porc. Roy. Irish. Acad. 54, 49

Payne, C.H., 1925, Stellar Atmospheres, Cambridge, Massachusetts

Russell, H.N., 1914, Popular Astronomy 22, 275

Russell, H.N., 1925a, Nature 116, 209

Russell, H.N., 1925b, Scientific American, October, 241

Russell, H.N., 1929, Ap. J. 70, 11

Russell, H.N., Dugan, R.S. and Stewart, J.S., 1927, Astronomy, Boston

Rutherford, E., 1937, The Newer Alchemy, Cambridge

Salpeter, E.E., 1952, Ap. J. 115, 326

Sandage, A.R. and Schwarzschild, M., 1952, Ap. J. 116, 463

Stratton, F.J.M., 1925, Astronomical Physics, London.

Strömgren, B., 1932, Zeitschr. f. Astroph. 4, 118

TAYLER: I think that you sounded unduly pessimistic about the state of stellar evolution in 1950. Speaking as someone who started research in stellar evolution in that year, it seems to me that the development of the subject from then onwards was relatively straightforward. In 1950 it was waiting for the arrival of the electronic computer. I was fortunate that very few papers were published in the subject, while I was doing my thesis, so that I did not need to read the journals. Then electronic computers got working and people like Martin Schwarzschild rapidly developed the subject. 